\title{
Improved Data Preprocessing Algorithm for Time-Domain Induced Polarization Method with Digital Notch Filter
}

\author{
Shuang-Chao $\mathrm{GE}^{1,2}$, Ming $\mathrm{DENG}^{2}$, Kai $\mathrm{CHEN}^{2}$, Bin $\mathrm{LI}^{3}$, and Yuan $\mathrm{LI}^{2}$ \\ ${ }^{1}$ North University of China, Taiyuan, Shanxi, China; e-mail: GeShCh@nuc.edu.cn \\ ${ }^{2}$ Key Laboratory of Geo-detection Ministry of Education, \\ China University of Geosciences, Beijing, China; \\ e-mail: dengming@cugb.edu.cn (corresponding author) \\ ${ }^{3}$ Technical Department TSLC, Taiyuan, Shanxi, China; e-mail: msepaper@163.com
}

\begin{abstract}
Time-domain induced polarization (TDIP) measurement is seriously affected by power line interference and other field noise. Moreover, existing TDIP instruments generally output only the apparent chargeability, without providing complete secondary field information. To increase the robustness of TDIP method against interference and obtain more detailed secondary field information, an improved dataprocessing algorithm is proposed here. This method includes an efficient digital notch filter which can effectively eliminate all the main components of the power line interference. Hardware model of this filter was constructed and Vhsic Hardware Description Language code for it was generated using Digital Signal Processor Builder. In addition, a time-location method was proposed to extract secondary field information in case of unexpected data loss or failure of the synchronous technologies. Finally, the validity and accuracy of the method and the notch filter were verified by using the Cole-Cole model implemented by
\end{abstract}

Ownership: Institute of Geophysics, Polish Academy of Sciences;

(C) $2016 \mathrm{Ge}$ et al. This is an open access article distributed under the Creative Commons Attribution-NonCommercial-NoDerivs license,

http://creativecommons.org/licenses/by-nc-nd/3.0/. 
SIMULINK software. Moreover, indoor and field tests confirmed the application effect of the algorithm in the fieldwork.

Key words: power line interference, digital notch filter, decay curve, apparent chargeability, SIMULINK.

\section{INTRODUCTION}

The induced polarization (IP) method was discovered more than 100 years ago by Conrad Schlumberger in 1913. The first measurements made using the IP method can be traced to the 1950s (Marshall and Madden 1959). After 60 years of development, two types of IP methods can now be used to collect data: spectral induced polarization (SIP) method and time-domain induced polarization (TDIP) method. TDIP measurement is characterized by low cost, high efficiency, and resistance to electromagnetic coupling. For these reasons, it has found wide use in mining exploration (Li et al. 2013), environmental and geohydrological detection (Gazoty et al. 2012, Martinho et al. 2006), and other geophysics detection applications. Hördt et al. (2007) estimated hydraulic conductivity in unconsolidated sediments from IP method to deduce the permeability. Dahlin et al. (2002) worked on the comparison between measurements with non-polarizable electrodes and stainless steel electrodes, and removed the spontaneous potential (SP) component from the record gained by the latter by subtracting the polarisation potential measured when no primary current and no IP signal were present. As a result, the observations cost of TDIP method could be cut down.

However, the disturbance rejection capability of existing TDIP instruments is insufficient. Use of TDIP is greatly limited by the effects of power line interference, various kinds of noises, and synchronous technology. Power line interference is a crucial source of noise in geophysical exploration with a fundamental frequency of 50 or $60 \mathrm{~Hz}$ (in China the fundamental frequency is $50 \mathrm{~Hz}$ ) and a multi-harmonic sinusoidal disturbance caused by an unknown memory less nonlinearity. To remove power line interference, almost all TDIP instruments made in China (Fan and Yang 1979, Feng and Fu 1994, Wu and Dou 1996, Zhang 2009) are implemented using a hardware twin-T filter, which is not sufficient to reduce strong interference to a satisfactory level, especially the harmonic components. Moreover, this filter has electronic circuit inertia which will lead to distortion of the measured wave (Deng and He 1998).

Researchers provided different techniques to reduce this interference, including adaptive methods (Dhillon and Chakrabarti 2001, Kohno et al. 1990, Wan et al. 2006), empirical mode-decomposition methods (Nimunkar and Tompkins 2007, Su and Chuang 2013), and wavelet transformation filters (Galiana-Merino et al. 2013, Sahambi et al. 1997). Most of the above- 
mentioned methods were designed for electrocardiograph (ECG) signal processing. Butler and Russell (1993) provided two methods to suppress the interference in the seismoelectric record by subtracting a noise estimate, in which block subtraction used an interval with obvious power line interference but negligible nonharmonic components as the subtrahend, and sinusoid subtraction used sinusoids with the parameters appropriated from the record as the subtrahend. The latter method was improved to be more applicable by overcoming the limitations of window length (Butler and Russell 2003), but there are still some limitations that the fundamental frequency must be very accurately known. Xia and Miller (2000) provided a hum filter by using damped least-squares Levinson-Marquardt inversion to approximate the amplitudes and phases of multiple harmonic components simultaneously. This technology was very effective for power line interference with stable amplitude and frequency, but the instability of the interference during the measurement for a specific trace would affect the effectiveness. It may not accurately be determined by power line noise in a pre-first arrival window if there are other sources of noise. Pankratov and Geraskin (2010) suppressed the industrial noise using a Finite Impulse Response (FIR) filter for SIP measurement, the phase delay of which was negligibly small compared to the typical anomalous value of the phase difference. But the suppression of the harmonics was not described in their work. Warden et al. (2012) used the curvelets technology to distinguish the interfering frequency from the signals, and results showed that it was a perfect time-frequency local analysis tool. But the premise was to select an appropriate mother wavelet.

These methods gained well application effect in geophysical data processing, especially for signal extraction and harmonic suppression in seismoelectric record. However, there are limitations on the execution of above-mentioned methods for their restrictions. And, their interference suppression capabilities and amplitude-frequency properties are not appropriate for TDIP requirements. Because the observation of TDIP is constantly changing electric field, it is inaccessible to obtain appropriate subtraction for the primary field and secondary field or accurately estimate the parameters of power line interference. Besides, the complex arithmetic of these filtering methods may affect real-time performance, making a simple and efficient notch filter essential. A notch filter has a certain stop-band width which could cope with the frequency fluctuation, and stop-band attenuation could be very large. As for the undesirable effects of the notch filter, the signal used in TDIP method generally has low frequency, so the disadvantage is negligible. Thus, we designed a digital notch filter to restrain the power line interference for TDIP method. It offers the advantage of simplicity and applicability, and could be helpful to make effective use of the dynamic range of the recording system. 
Another issue is that most of the previous TDIP measurements considered only polarizability and chargeability, and only some discrete data in small windows were collected, resulting in an incomplete description of the observed voltage decay. However, the IP decay curve contains much more information than chargeability (Tong et al. 2006). In addition, the shape of the IP decay curve contains spectral information of the IP phenomenon that can be extracted only by using the full decay in the inversion process (Fiandaca et al. 2012). Moreover, observation of entire time series is necessary to resolve the Cole-Cole parameters accurately (Swift Jr. 1973, Hönig and Tezkan 2007).

To address these deficiencies and shortcomings, an improved dataprocessing algorithm with a digital notch filter is proposed here. The notch filter is a FIR filter, easy to be implemented in hardware. Comparison tests showed that the parameters calculated by using the raw data without filtering were easily distorted by ambient noise and synchronization exception, but the results yielded by using the notch filter were significantly better than that without filtering, and more accurate to the theoretical values than the results obtained by using other filtering method. In practical tests, the complete decay curve and apparent chargeability were obtained in the situation of strong interference and synchronization failure by using the algorithm with this notch filter. A complete decay curve gives access to new applications of TDIP in environmental and hydrogeophysical investigations by extracting more detailed relaxation time spectrum and IP transients directly from TDIP (Tong et al. 2006, Fiandaca et al. 2012). This paper includes a detailed introduction to the digital notch filter design and modeling, the secondary field time-orientation algorithm, and de-noising algorithms. Finally, the effectivity of the filter and data-processing method were tested by a series of tests.

\section{THEORY OF THE TDIP METHOD}

Usually, TDIP uses rectangular current pulses of opposite polarity as an excitation source, in which pulses and pauses of the same duration are used with a $50 \%$ duty cycle (Fig. 1a). Immediately after the current is turned on, an induced potential, $\Delta V_{2}$, is raised across the potential electrodes. After a charge-up effect, the primary voltage, $\Delta V_{1}$, is measured just before the current is turned off to compute the direct current (DC) resistivity. When the current is turned off, the voltage drops to a secondary level and then decays with time during the relaxation period (Fig. 1b). 

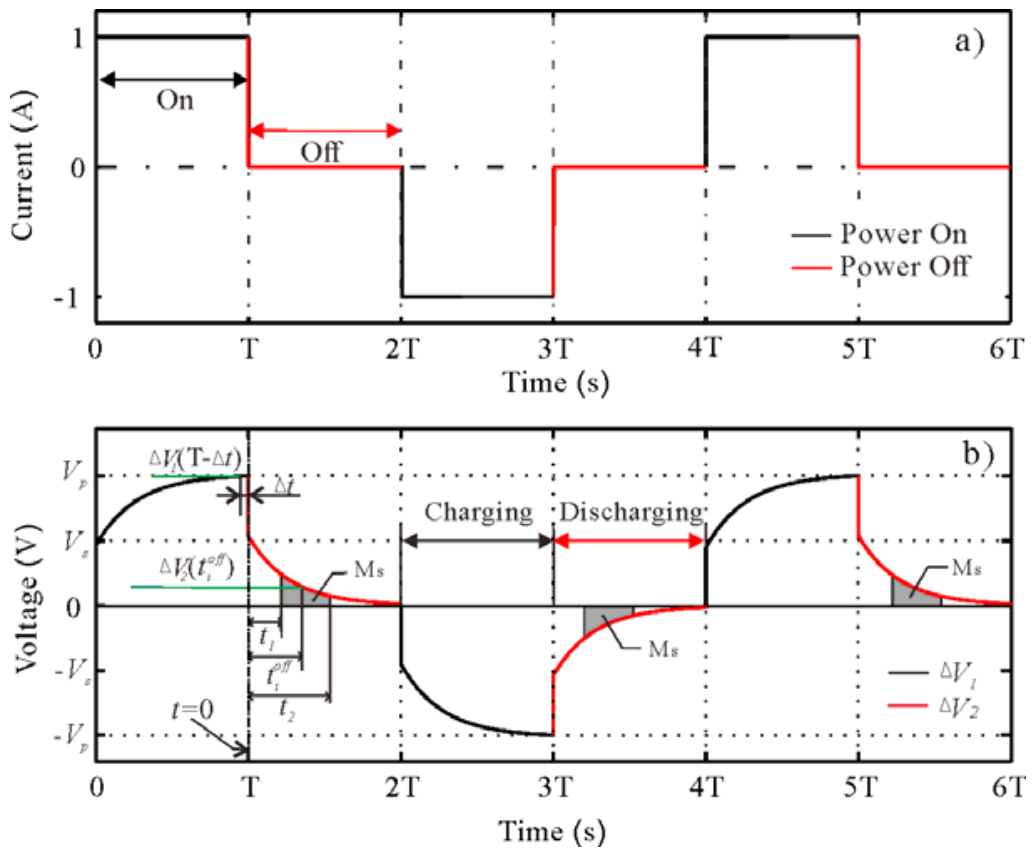

Fig. 1. The schematic of TDIP method: (a) excitation source for TDIP; (b) voltage curve for TDIP. The black and red line in panel (a), respectively, represents poweron and power-off period. The curves of the same colors in panel (b) are corresponding to primary field and secondary field. Some key time nodes are marked in (b).

The TDIP method measures the voltage decay induced by turning off the exciting current pulse and uses the characteristics of the decay to study the induced polarization, also known as time-domain chargeability. Seigel (1959) defined chargeability $M_{a}$ as:

$$
M_{a}=V_{s} / V_{p}
$$

where $V_{s}$ is the secondary field voltage immediately after the current is shut off and $V_{p}$ is the effective primary voltage. In China, the apparent chargeability, $\eta_{s}$, is most commonly used:

$$
\eta_{s}=\frac{\Delta V_{2}\left(t_{i}^{\text {off }}\right)}{\Delta V_{1}(T-\Delta t)} \times 100 \%
$$

where $t=0$ is the time node when the excitation current is turned off; $t_{i}^{\text {off }}$ is a lapse time with respect to $t=0$, typically close to $0.5 \mathrm{~s}$; and $\Delta V_{2}\left(t_{i}^{\text {off }}\right)$ is the voltage of the secondary field at this time; $\Delta V_{1}(T-\Delta t)$ is the total field 
voltage, where $T$ is the charging time, and $\Delta t$ is a very short time before the excitation current is turned off so that the total field voltage is close to saturation voltage.

The precision of $\eta_{s}$ is defined by $\Delta V_{2}\left(t_{i}^{\text {off }}\right)$. However, the secondary voltage is difficult to measure accurately in the field, and as a result, modern IP instruments no longer measure the instantaneous value of $\Delta V_{2}\left(t_{i}^{\text {off }}\right)$, but the mean value over the time window $\left[t_{1} t_{2}\right], \frac{\int_{t_{1}}^{t_{2}} \Delta V_{2}(t) d t}{\left(t_{2}-t_{1}\right)}$. Therefore, another expression of apparent chargeability $M_{s}$ can be expressed as:

$$
M_{s}=\frac{\int_{t_{1}}^{t_{2}} \Delta V_{2}(t) d t}{\left(t_{2}-t_{1}\right) \cdot \Delta V_{1}(T-\Delta t)} \times 100 \%
$$

where, $t_{1}$ and $t_{2}$ are lapse times with respect to $t=0$ (as shown in Fig. 1b). According to the Newmont standard, $t_{1}=0.45 \mathrm{~s}$ and $t_{2}=1.1 \mathrm{~s}$ (Swift Jr. 1973).

\section{DATA-PROCESSING ALGORITHM}

Pankratov and Geraskin (2010) put forward some reference processing algorithms for IP data processing including detrend and distortion suppression. However, their methods were designed for SIP method, and mainly focused on frequency-domain characteristics. To suppress the interference and obtain more complete IP information in time-domain, a data-processing algorithm is proposed in this paper (Fig. 2). Using a digital notch filter, segmentation, and overlapping averaging, a TDIP decay curve and apparent chargeability can be obtained. As part of this process, several key issues must be addressed: an efficient digital notch filter, exact secondary-field time-range determination, random noise suppression, SP removal technology, IP decaycurve extraction, and the apparent chargeability calculation.

\subsection{Digital notch filter}

According to the characteristic of the power line interference that its energy is concentrated mainly on the fundamental frequency and the odd harmonics, the zero location of the new notch filter should be as in Fig. 3, where $\omega_{0}=2 \pi f_{0} / f_{s}$, and $f_{0}$ is the fundamental frequency and $f_{s}$ is the sampling rate. For TDIP measurement, $f_{s}$ was commonly $120 \mathrm{~Hz}$. But with the increase of the system's throughput, $f_{s}$ is raised to $2400 \mathrm{~Hz}$.

The approach used here to design this notch filter is first to design a comb filter and then to implement a notch filter by taking an all-pass filter and subtracting the comb filter. The all-pass filter, which is intended for 


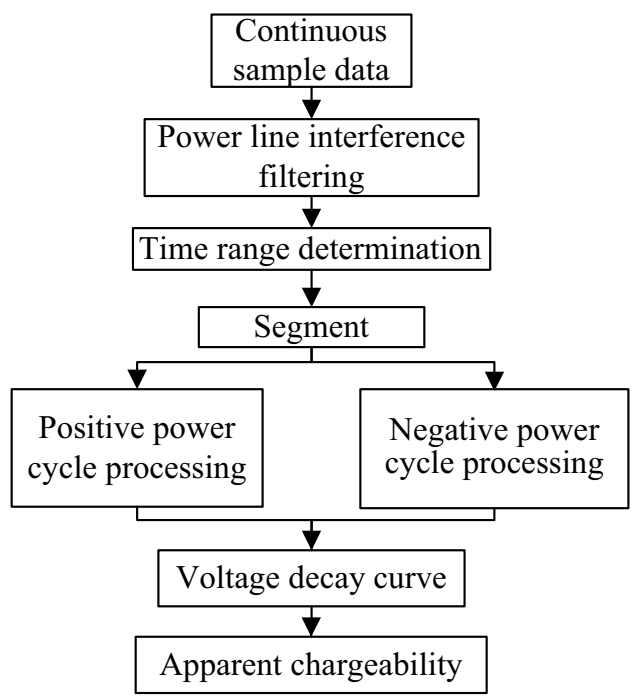

Fig. 2. TDIP measurement algorithm flowchart.

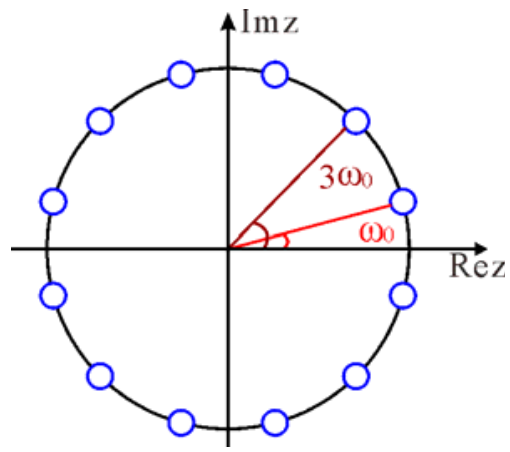

Fig. 3. Zero locations of the notch filter. The blue cycles represent the angular frequency of the odd harmonics. The red line represents the angular frequency $\omega_{0}$ of the fundamental and the brown line represents $3 \omega_{0}$ of the third harmonic, and so on.

signal delay purposes, is used to cancel the phase delay caused by the comb filter.

The comb filter is designed based on a narrow-band band-pass filter proposed by $\mathrm{Hu}$ (2003). The amplitude-frequency characteristic of this bandpass filter can be expressed as:

$$
H_{b p}(z)=\frac{1+z^{-M}}{\left(1-z^{-1} e^{j \omega_{0}}\right)\left(1-z^{-1} e^{-j \omega_{0}}\right)}
$$


where $\omega_{0}=2 \pi f_{0} / f_{s}, j$ is a square root of -1 , and $z$ is the delay unit which is equal to $e^{j \omega}$; and $M$ is the order of the comb filter, in other words, the number of the comb filter poles.

From Eq. 4, the filter permits only frequencies close to the fundamental frequency to pass. Therefore, the comb filter is designed by setting poles at odd harmonic frequencies, $f_{\text {odd }}=(2 i-1) \omega_{0}, i=1,2, \ldots, n / 2$, and $\left.n=f_{s} / f_{0}\right)$ (Fig. 3). Hence, the comb filter amplitude-frequency characteristic is:

$$
H_{c f}(z)=\frac{1+z^{-M}}{\prod_{i=1}^{\frac{n}{4}}\left[\left(1-z^{-1} e^{\mathrm{j}(2 i-1) \omega_{0}}\right) \times\left(1-z^{-1} e^{(-1) \times \mathrm{j}(2 i-1) \omega_{0}}\right)\right]}=\frac{1+z^{-M}}{1+z^{-n / 2}}
$$

Because only the odd harmonics are considered, the scope of $i$ is from 1 to $n / 4$. There is no odd harmonic interference except the fundamental frequency in the Nyquist band when $n<4$. In that case, general band-stop filter could be used to deal with the interference. Winder (2002) described the design method of the band-stop filter in detail, so we will not cover it again.

The comb filter is designed based on pole zero cancellation method, so the fundamental pole $\omega_{0}$ is also the zero point of the filter. Thus, we substitute $\omega_{0}=2 \pi f_{0} / f_{s}$ into $z=e^{-j M \omega_{0}}$. And next substituting $z$ into the numerator of Eq. 5 gives:

$$
1+e^{-j 2 \pi M \frac{f_{0}}{f_{s}}}=0 \Rightarrow \mathrm{e}^{-j 2 \pi M \frac{f_{0}}{f_{s}}}=-1 \Rightarrow j 2 \pi M f_{0} / f_{s}=(2 d+1) \pi \Rightarrow M=(2 d+1) \times n / 2,
$$

where $d$ is an integer. So, the frequency response of this comb filter is:

$$
\begin{aligned}
H_{c f}\left(e^{j \omega}\right)= & \frac{1+e^{-j \omega M}}{1+e^{-j \omega \frac{n}{2}}}=\frac{1+\cos (\omega M)+j \sin (\omega M)}{1+\cos \left(\frac{n \omega}{2}\right)+j \sin \left(\frac{n \omega}{2}\right)} \\
& =\frac{2\left[\cos \left(\frac{\omega M}{2}\right)\right]^{2}+j 2 \cos \left(\frac{\omega M}{2}\right) \sin \left(\frac{\omega M}{2}\right)}{2\left[\cos \left(\frac{\omega n}{4}\right)\right]^{2}+j 2 \cos \left(\frac{\omega n}{4}\right) \sin \left(\frac{\omega n}{4}\right)} \\
& =e^{-j \omega\left(\frac{M}{2}-\frac{n}{4}\right)} \frac{2 \cos \left(\frac{\omega M}{2}\right)}{2 \cos \left(\frac{\omega n}{4}\right)}=e^{-j \omega d \frac{f_{s}}{2 f_{0}}} \frac{2 \cos \left[(2 d+1) \frac{\omega f_{s}}{4 f_{0}}\right]}{2 \cos \left(\frac{\omega f_{s}}{4 f_{0}}\right)}
\end{aligned}
$$

According to the nature of the trigonometric function, the extreme value of Eq. 7 is $(2 d+1)$. So Eq. 5 is normalized to: 


$$
H_{c f}(z)=\frac{1+z^{-(2 d+1) \frac{f_{s}}{2 f_{0}}}}{(2 d+1) \times\left(1+z^{-\frac{f_{s}}{2 f_{0}}}\right)}
$$

The corresponding all-pass filter can be expressed as:

$$
H_{a p}(z)=z^{-d \frac{f_{s}}{2 f_{0}}} .
$$

Finally, the required notch filter is calculated by subtracting the comb filter from the all-pass filter as follows:

$$
H_{n f}(z)=z^{-d \frac{f_{s}}{2 f_{0}}}-\frac{1+z^{-(2 d+1) \frac{f_{s}}{2 f_{0}}}}{(2 d+1) \times\left(1+z^{-\frac{f_{s}}{2 f_{0}}}\right)}
$$

To achieve better amplitude-frequency characteristics and reduce ripple, a multi-level filter ( $k$-order) is used, and therefore Eq. 10 becomes:

$$
\begin{aligned}
& H_{n f}(z)=\left(z^{-d \frac{f_{s}}{2 f_{0}}}\right)^{k}-\left[\frac{1+z^{-(2 d+1) \frac{f_{s}}{2 f_{0}}}}{(2 d+1) \times\left(1+z^{-\frac{f_{s}}{2 f_{0}}}\right)}\right]^{k}=\left(z^{-d \frac{f_{s}}{2 f_{0}}}\right)^{k}-\left\{\frac{1-\left(-z^{-\frac{f_{s}}{2 f_{0}}}\right)^{(2 d+1)}}{(2 d+1) \times\left[1-\left(-z^{-\frac{f_{s}}{2 f_{0}}}\right)\right]}\right\}^{k} \\
& =\left(z^{-\frac{f_{s}}{2 f_{0}}}\right)^{d k}-\left[\frac{1}{(2 d+1)} \sum_{i=0}^{2 d}\left(-z^{-\frac{f_{s}}{2 f_{0}}}\right)^{i}\right]^{k}
\end{aligned}
$$

As a result, we developed a multi-frequency notch-filter with FIR structure which could suppress all of the odd harmonics and the fundamental frequency. An example is shown in Fig. 4 , with $f_{s}=1000 \mathrm{~Hz}$ and $f_{0}=50 \mathrm{~Hz}$. This notch-filter's amplitude-frequency characteristic is determined by $d$ and $k$ (Fig. 5).

As shown in Figs. 4 and 5, $d$ permits main control of the stop-band characteristics including the attenuation amplitude and the bandwidth, the bigger the $d$ the smaller the attenuation and the narrower the stop-band width, and $k$ mainly determines the ripple of the passband, the bigger the $k$ the smaller the ripple. Specifically, the relation of the above-mentioned indexes with $k$ versus $d$ is shown in Fig. 6. 


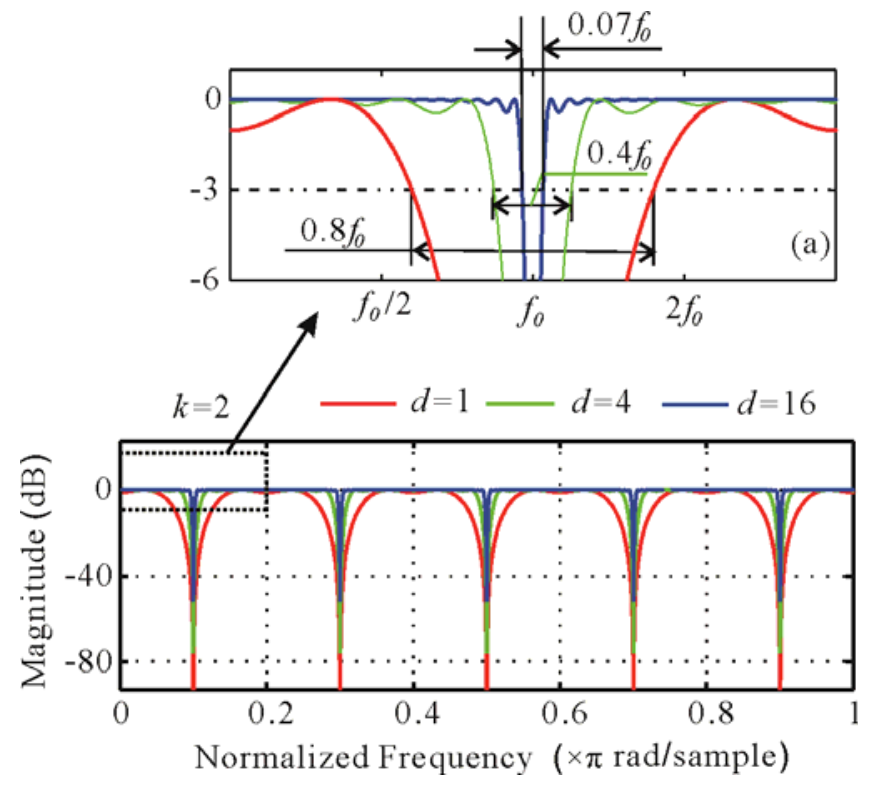

Fig. 4. Frequency response of the notch filter with fixed $k$. Panel (a) is zoomed image of the section within the dotted box and it shows the bandwidth of the stop-band at $-3 \mathrm{~dB}$.

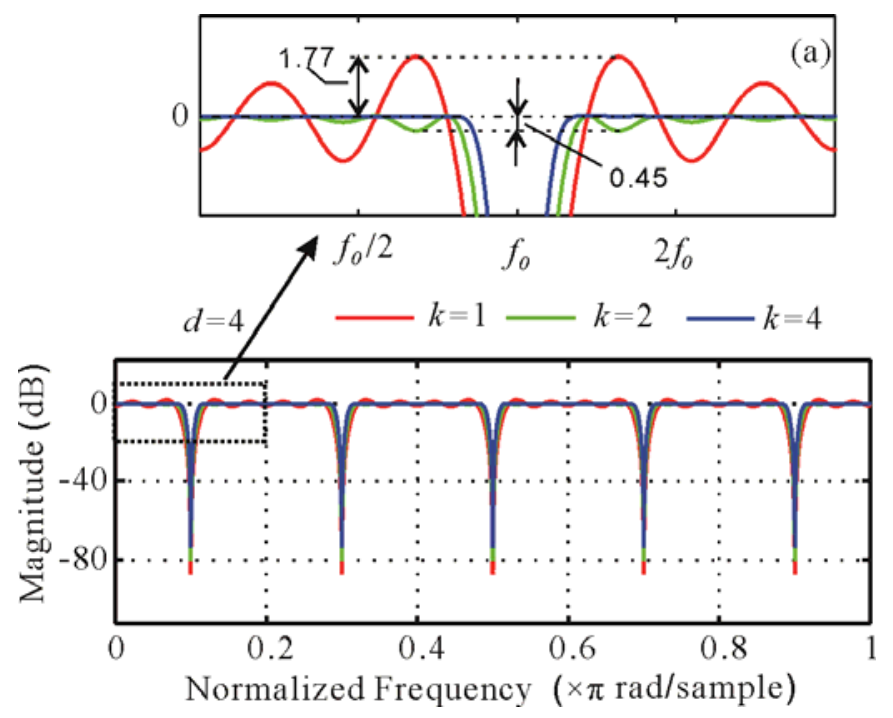

Fig. 5. Frequency response of the notch filter with fixed $d$. Panel (a) is zoomed image of the section within the dotted box and it shows the ripple strength of the passband. 

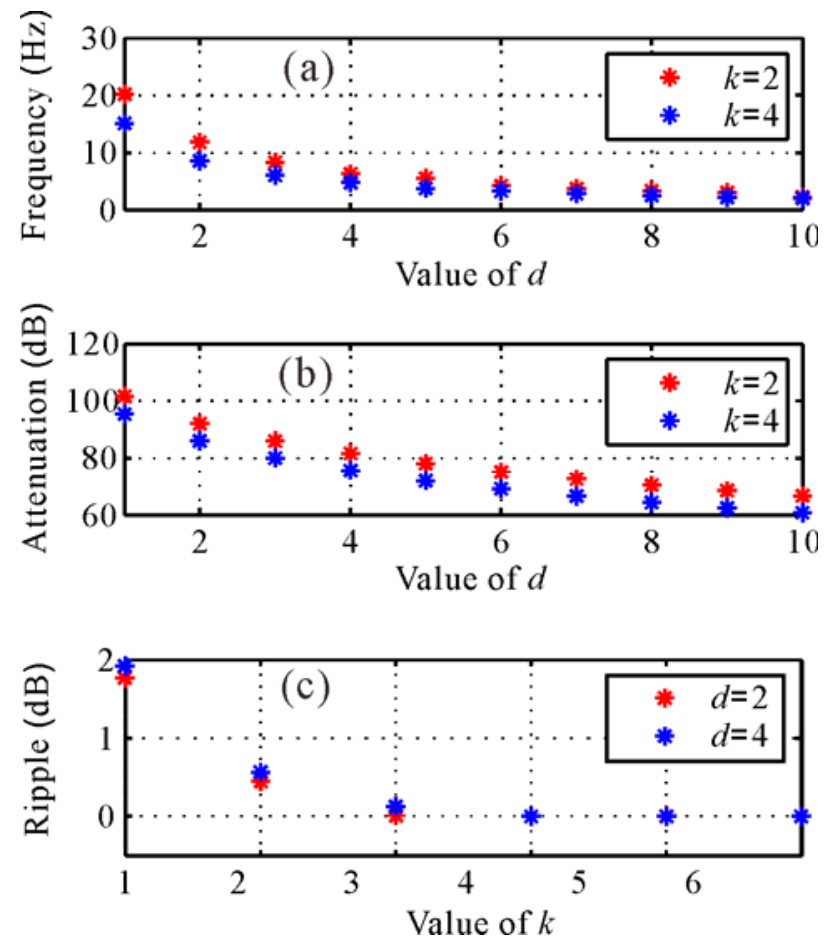

Fig. 6. Impact of the key parameters on the indexes of the notch filter. Panels (a) and (b): impact of $d$ on the indexes of the notch filter with fixed $k$; (a) the bandwidth at $3 \mathrm{~dB}$; (b) the attenuation amplitude at the fundamental frequency; (c) impact of $k$ on the ripple of the notch filter with fixed $d$.

The choice of $d$ should take into account of the interference suppression and undesirable effects of the notch filter. And $k$ is not the bigger the better, because the length of the filter will increase exponentially with $k$ and $d$, especially $k$, which leads to a rapid increase of hardware resource consumption. After all these comprehensive consideration, $d$ and $k$ were suggested as 4 and 2 for the notch filter used in TDIP. This notch filter has no lowfrequency decay and phase delay, and all odd harmonics can be filtered with stop-band attenuation $\approx 80 \mathrm{~dB}$. In addition, a certain stop-band width can deal with small fluctuations in the power line interference. This is a FIR filter with the advantages of stability and linear phase. The filter was implemented in both $\mathrm{C}++$ code and MATLAB code so that it could be used in various situations. Furthermore, a hardware model of the filter was constructed in Digital Signal Processor (DSP) Builder (Fig. 7). DSP Builder is a product for performing Model-Based Design targeting Altera Field Programmable Gate Array (FPGA). By using Altera DSP Builder, algorithm 


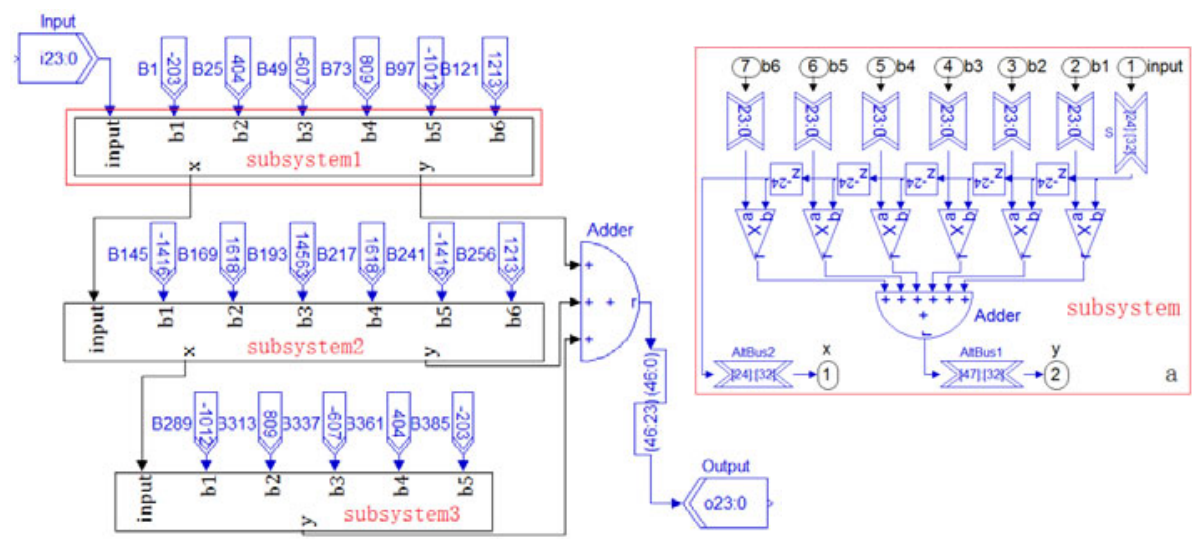

Fig. 7. Hardware model of the notch filter. $f_{s}=2400 \mathrm{~Hz}$ and $f_{0}=50 \mathrm{~Hz}$. The input data and output data are adjustable, and we set them to 24 bit here. Panel (a) is the internal structure of the subsystem.

and system designers can shorten DSP design cycles by creating the hardware representation of a DSP design in an algorithm-friendly development environment as well as share a common development platform. We utilized the DSP builder to implement a sample project for the filter and then exported it to the FPGA and compiled in Vhsic Hardware Description Language (VHDL) to obtain an intellectual property core (IP core) which could be integrated into the host. Beyond TDIP data processing, this notch filter can also be applied to other electromagnetic prospecting problems.

\subsection{Noise reduction and SP removal}

In addition to the power line interference, random noise and SP can also severely interfere with TDIP measurement. Because in this method TDIP data are collected for several successive cycles, it is possible to improve the signal to noise ratio (SNR) using segmentation and digital averaging.

Before segmentation, the time range of each power cycle should be determined. In most cases, synchronous technologies can provide an accurate temporal location (Deng et al. 2006), but they will not work properly under adverse circumstances. To overcome this limitation, an evaluation method for time location was proposed, called the "shifting average difference method (SAD)", as described below.

The first step is to determine the shift number $N_{\text {order }}$. This parameter is determined by the nature of the notch filter used in the process, and the filter program in Section 3.1 will automatically produce it. Hence, a $n_{\text {order-point }}$ 
moving-average filter $\left(n_{\text {order }}=N_{\text {order }}-1\right)$ is first used to filter sequence $x$ starting at the first data point to obtain $x_{1}$, where $x$ is the previous segment (length is $T f_{s}$, where $T$ is the charging time) of the data processed by the notch filter. Second, the first-order difference of $x_{1}$, denoted by $\mathbf{d} \mathbf{x}_{1}$, is calculated. The next step is to find the location of the maximum absolute value of $\mathbf{d} \mathbf{x}_{1}$, denoted by $t_{1}$. Then one-step left shift is performed for $x$ each time, and the above three steps are repeated. After norder iterations, the corresponding $t_{2}, t_{3}, \ldots, t_{\text {Norder }}$ are calculated. And then the first-order difference of the sequence $\left\{t_{1}, t_{2}, t_{3}, \ldots t_{N o r d e r}\right\}$ is calculated, denoted by $\mathbf{d}_{\mathbf{t}}$. The location where $\mathbf{d}_{\mathbf{t}}$ begins to be convergent is denoted by $c$, and $t_{c}$ is the jump edge. Based on comprehensive consideration of certain constraint conditions, including the jump-edge directionality of different power cycles and the periodicity of the secondary field, the initial time of the first secondary field, denoted by $t_{s f 1}$, can be obtained. Finally, using $t_{s f 1}, f_{s}$, and the period for each power cycle, the time information of the primary and secondary fields can be obtained. An algorithm flowchart of this method is shown in Fig. 8. To implement this procedure, it was coded in $\mathrm{C}++$ and in MATLAB, respectively, generating two different but equivalent codes to suit different applications.

On this basis, the filtered data are segmented by positive or negative power cycle according to the time information calculated earlier, and then the positive segments are overlaid and averaged, as well as the negative segments. During this process, the useful signal is stacked in the same phase, but the interference components are stacked in different phases, further increasing SNR. This method yields two charge-discharge curves with opposite power direction. For SP, existing IP instruments usually combine manual

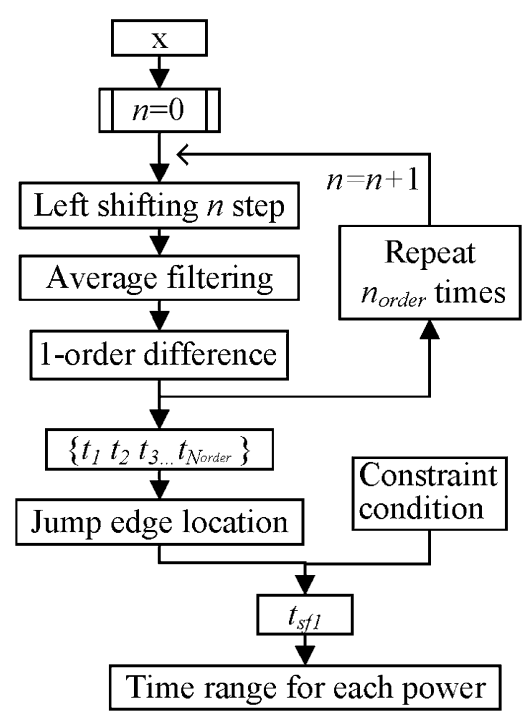

Fig. 8. Flowchart of time location. $t_{s f 1}$ is the initial time of the first secondary field. 
and automatic compensation. This method will impact the amount of useful information in the presence of residual $\Delta V_{2}$. Using the DC feature of SP, the charge-discharge curves of the positive and negative power cycles are averaged to remove SP from the secondary field. Finally, the secondary field decay curve can be obtained. In this way, random noise is decreased, and SP is also removed.

\subsection{Decay curve and apparent chargeability}

The approach described above yields a voltage decay curve for a complete power cycle. To improve the reliability of the results, the test values of repeated measurements can be averaged. Thus, a complete TDIP decay curve is obtained, composed of a complete primary field $\Delta V_{1}$ curve and secondary field $\Delta V_{2}$ curve.

In addition, the apparent chargeability $\eta_{s}$ at different delay times can also be obtained. To do this, the average values of $\Delta V_{2}$ in a time box (adjustable) can be calculated as a delay of the increase in the decay curve and then divided by the average value of the second half of $\Delta V_{1}$ curve. In this way, the apparent chargeability at different points in time can be obtained.

\section{RESULTS}

\subsection{Simulation test}

The method described above was verified using the Cole-Cole model implemented in Simulation and Link (SIMULINK). SIMULINK, developed by MathWorks, is a graphical programming environment and widely used in automatic control and digital signal processing for multi-domain simulation and Model-Based Design. Models are represented graphically in SIMULINK as block diagrams. One of the primary advantages of employing SIMULINK for the analysis of dynamic systems is that it allows us to quickly analyze the response of complicated systems that may be prohibitively difficult to analyze analytically.

In general, the mathematical equations representing a given system that serve as the basis for a SIMULINK model can be derived from physical laws. In this section, we derived a mathematical model for TDIP measurement and then implemented that model in SIMULINK, as shown in Fig. 9. The supply current consisted of three parts: $I=I_{0}+I_{n}+I_{s p}$, where $I_{0}$ was a forward and reverse pulse, 2 A with 1:1 duty cycle, with each power supply lasting $2 \mathrm{~s} ; I_{n}$ was a fixed amount of random noise and power line interference with a fundamental frequency of $50 \mathrm{~Hz}$, and $I_{s p}$ was DC (equivalent of SP). The sampling rate was $2400 \mathrm{~Hz}$, and the total sampling time was $48 \mathrm{~s}$. The first step was to use $I$ as an input stimulus current and the raw voltage data were acquired (shown in Fig. 10a). Obviously, the useful signal was 


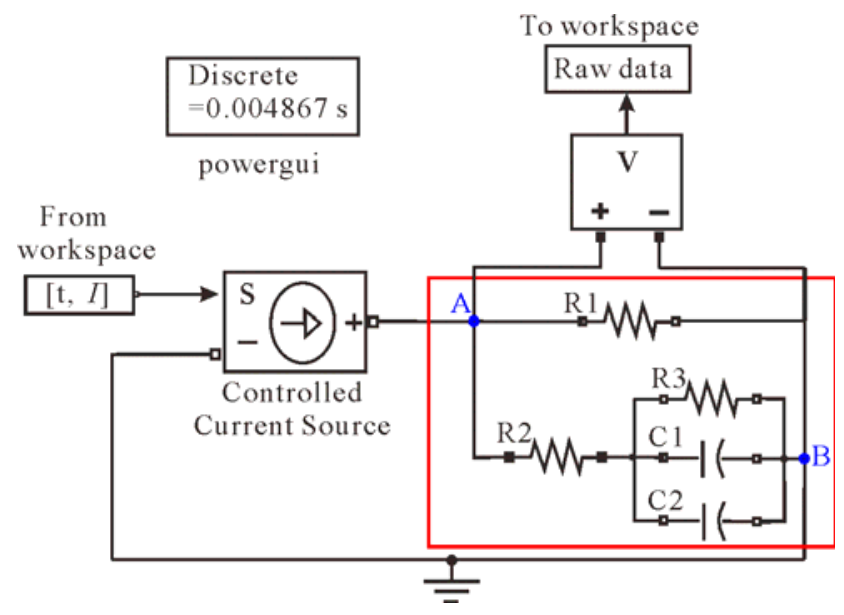

Fig. 9. SIMULINK model for TDIP measurement. The sample data was the potential difference between points A and B. The TDIP result was a reflection of the IP effect of the model in the red box, where $\mathrm{R} 1=10 \Omega, \mathrm{R} 2=40 \Omega, \mathrm{R} 3=80 \Omega$, and $\mathrm{C} 1=\mathrm{C} 2=5 \mathrm{E}-3 \mathrm{~F}$.

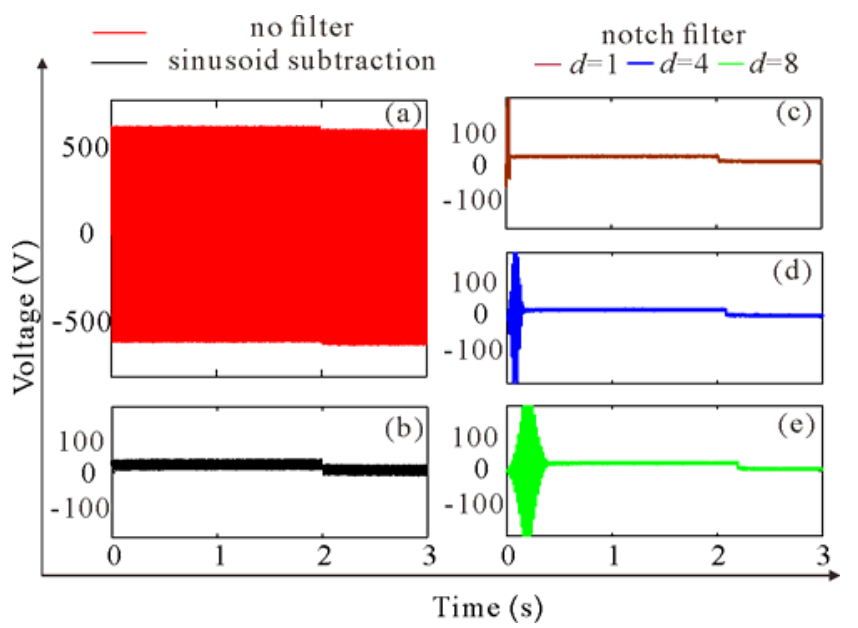

Fig. 10. Time-domain waves. Parameter $k$ for the three notch filters was fixed as 2 , and $b$ was variable for filter performance analysis.

completely submerged by the powerful power line interference, and therefore the top priority was to remove this interference. The raw data were first filtered using two methods: (i) method 1: sinusoid subtraction (other filtering methods were not discussed due to their algorithmic complexity or precondition), as shown in Fig. 10b; and (ii) method 2: the notch filter described in Section 3.1 with $k=2$ and $d=1,4,8$, as shown in Fig. 10c-e. 


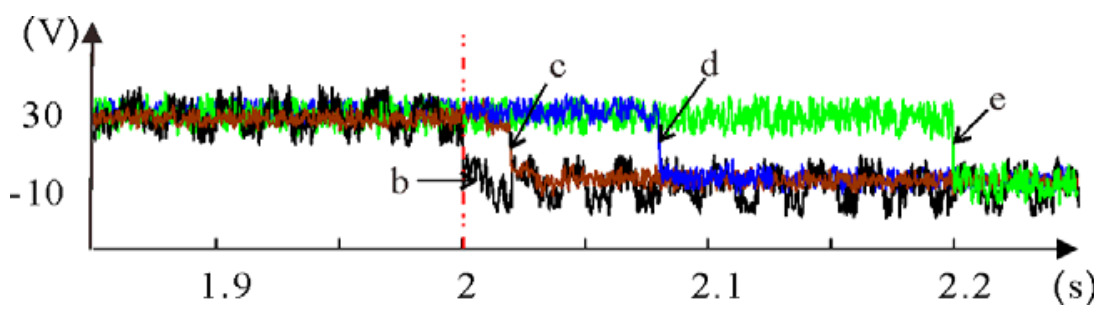

Fig. 11. Detail description of the time-domain data: panels (b) through (e) show the same curves as Fig. 10b-e.

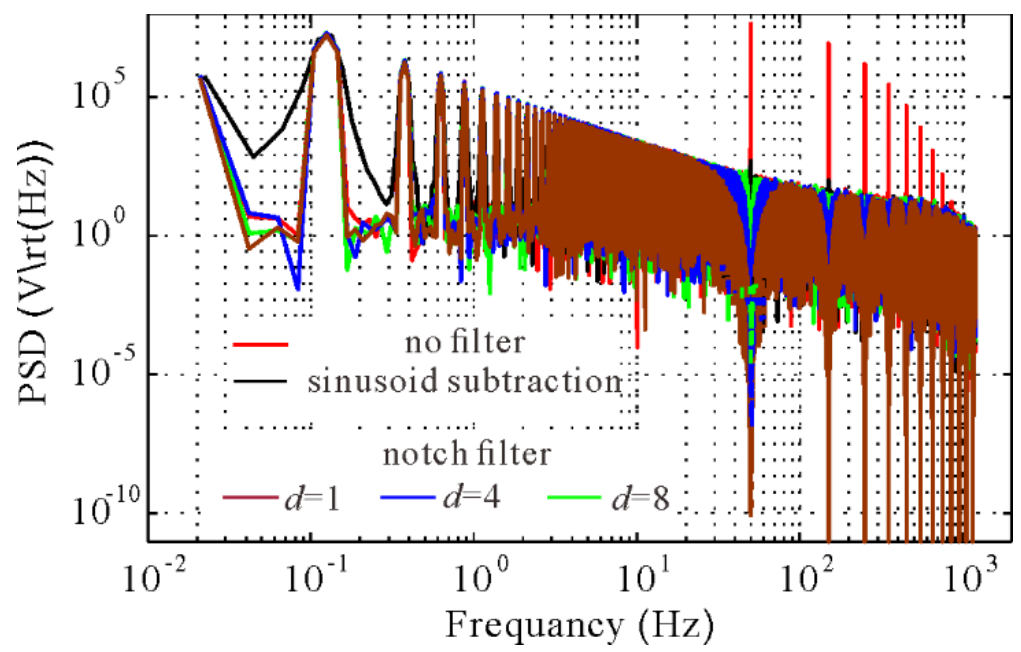

Fig. 12. Frequency-domain waves. The PSD were calculated by Fast Fourier Transform (FFT) algorithm, with length of 115200 and hanning window.

As shown in Fig. 10, with the increase of $b$, the nonstationary process was extended and the filtering effect reduced. But too small $\mathrm{d}$ would lead to boundary confusion within the primary field and the secondary field, as shown in Fig. 11. So, $k=2$ and $d=4$ were selected as the optimal paremeters for the notch filter. As for sinusoid subtraction, this approach has the virtue of early stability and no phase delay. But its interference suppression ability appeared to be weaker than the notch filter and it could not cope with the frequency fluctuation of the interference, as shown by the Power Spectral Density (PSD) curves in Fig. 12.

By analyzing the frequency spectra of the time-domain data (Fig. 12), it becomes clear that the notch filter has successfully removed the power line interference while preserving the remaining spectral content of the excitation signal. The next step was to extract the time information of each power cycle using the SAD method. Next, the random noise and SP mixed into the fil- 

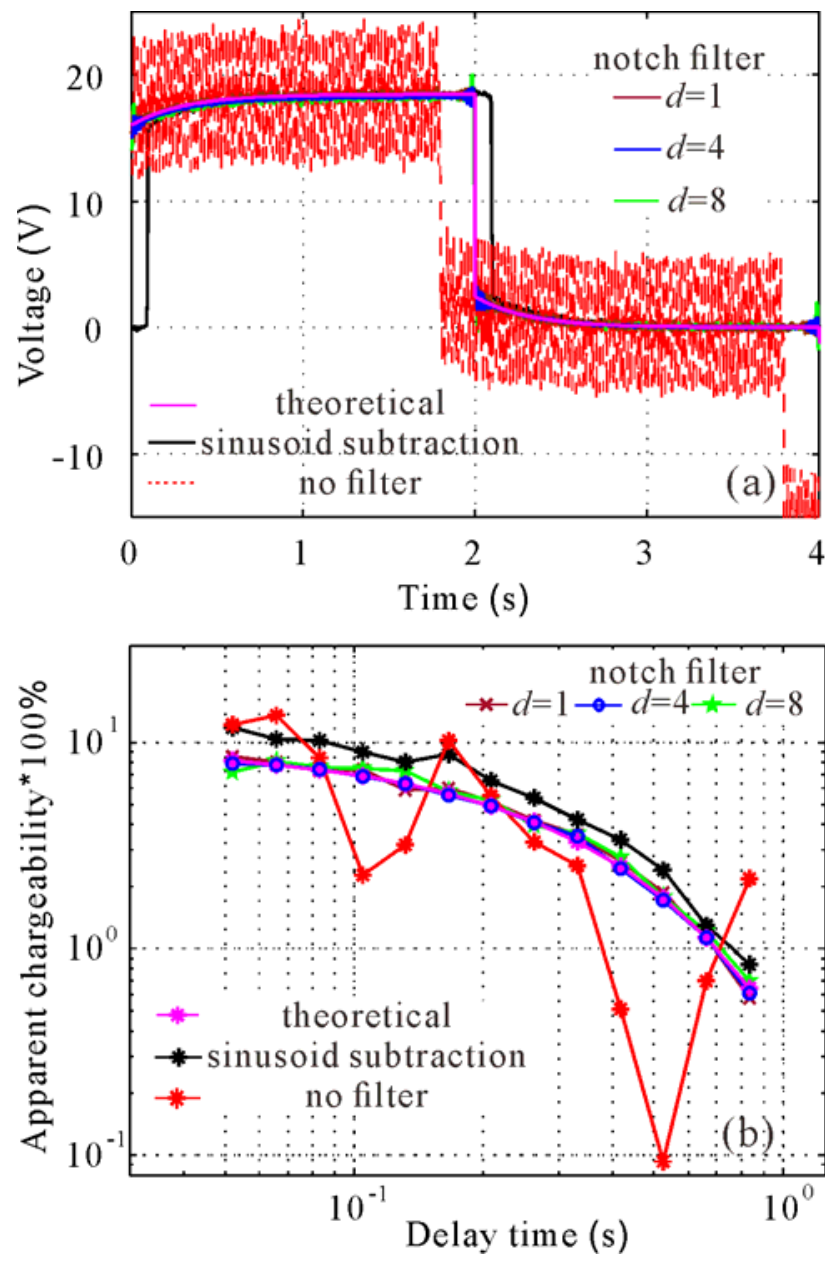

Fig. 13. Simulation results: (a): TDIP voltage decay curves; (b) comparison of the apparent chargeability gained by different methods. The pink line in panel (a) is the theoretical decay curve of this model which is collected by using $I_{0}$ as the excitation signal. The apparent chargeability curves in different colors in panel (b) are corresponding to the decay curves of the same color in (a).

tered data $x$ were processed. Finally, after low-pass filtering and calculation, the model's charge-discharge curve was obtained (Fig. 13a) as well as the apparent chargeability $\eta_{s}$ (Fig. 13b).

The simulation results show that the secondary field information calculated by using the selected notch filter is closer to the theoretical values, which proves the accuracy and effectiveness of the filter and the proposed method. 
To simulate adverse circumstances under which the synchronous technologies cannot work well or data loss may occur (Deng et al. 2008), a random piece of data was removed from the actual data set at the beginning of the complete time series. In this case, the SAD method could determine the time range of the secondary field exactly. Therefore, the effective decay curve and the apparent chargeability could still be obtained.

\subsection{Indoor test}

Indoor test was carried out on the campus lawn at China University of Geoscience (Beijing). The charging and discharging time was both $2 \mathrm{~s}$ and power cycle was $8 \mathrm{~s}$, with the emission current $5 \mathrm{~A}$ and $f_{s}$ of $2400 \mathrm{~Hz}$. The tests lasted about $30 \mathrm{~min}$ and three-channel data (with the electrodes parallel with each other) for 40 power cycles were collected. A segment of the raw data was shown in Fig. 14.

During this experiment, there was a lag time about $0.09 \mathrm{~s}$ between the receiver and the transmitter due to some hardware malfunction, as shown in Fig. 14 marked by terror. Traditional method and the new method were used in data processing, and the decay curve and chargeability were shown in Figs. 15 and 16.

The delay time brought a mismatch between the results calculated by two different methods. The starting time of the secondary field in the traditional method was earlier than the actual value $(2 \mathrm{~s})$. But the new algorithm got the correct time information for the decay curve.

The results of the three channels were about the same, so the curves with same symbols but different colors were overlapped on each other. But there were large differences between the results obtained by the two methods.

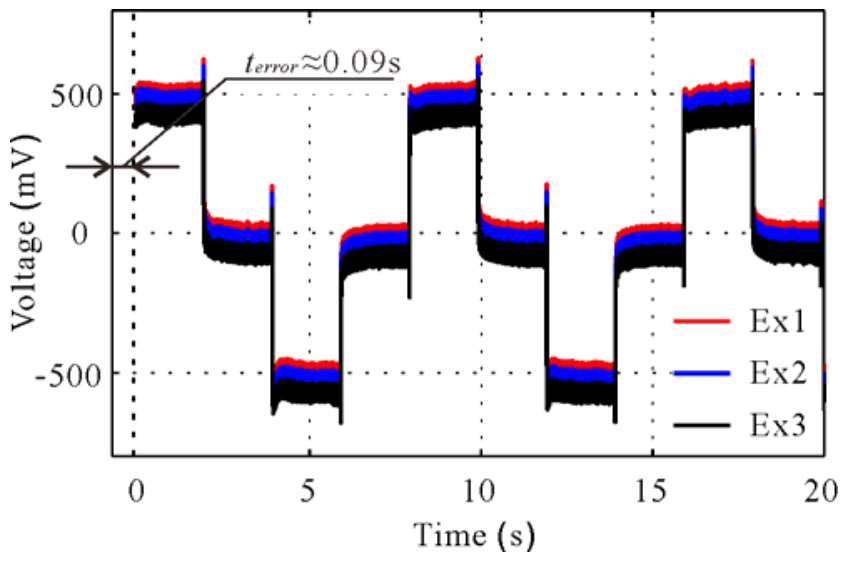

Fig. 14. Raw data of the lawn test. Colors: red, blue, and black represent the data collected by channel 1, channel 2, and channel 3 (Ex1, Ex2, and Ex3). 


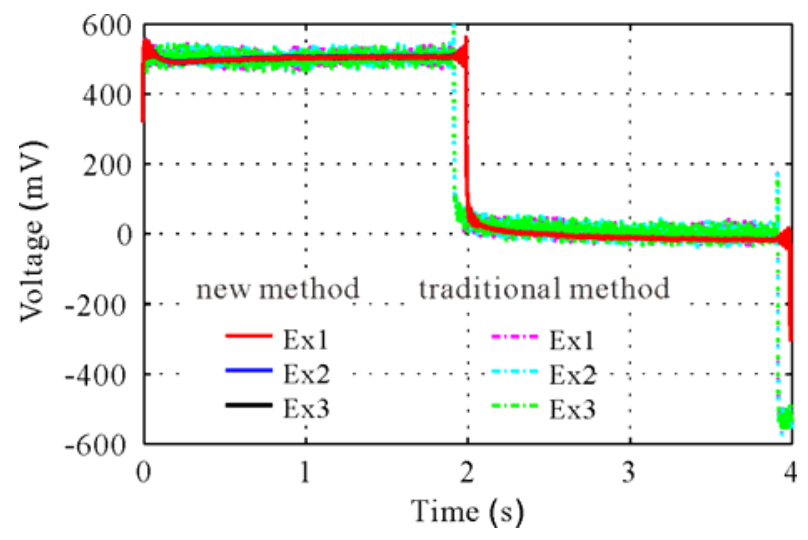

Fig. 15. Decay curve of the lawn test. The solid curves were calculated by the new method, and the dotted curves were analogue results of the traditional method for comparison. The traditional method could not output complete decay curve, because that it just collected partial data of the secondary field. Colors: red, blue, and black represent the results obtained by the data collected by channel 1 , channel 2 , and channel 3.

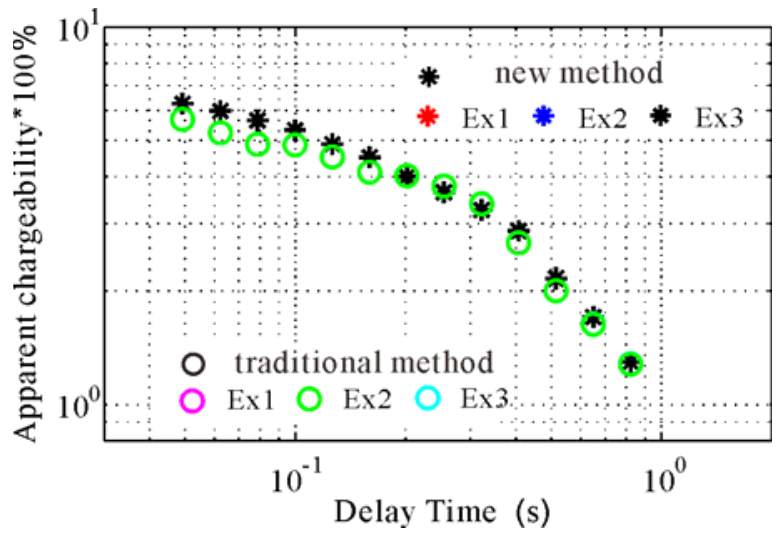

Fig. 16. Apparent chargeability of the indoor test. The curves of red, blue, and black represent the results obtained by the data collected by channel 1 , channel 2 , and channel 3. Symbols: results obtained by using traditional method (o) and the dataprocessing algorithm proposed in this paper $(*)$, respectively.

\subsection{Field test}

Field tests were conducted in Xilin Gol Grassland, Inner Mongolia, which is a sparsely populated region. Results were shown in Fig. 17.

The spikes in Fig. 17 were caused by over-charging. They have seriously affected the observations, so we removed these abnormal data by using curve fitting, similar to the approach taken in Pankratov and Geraskin 


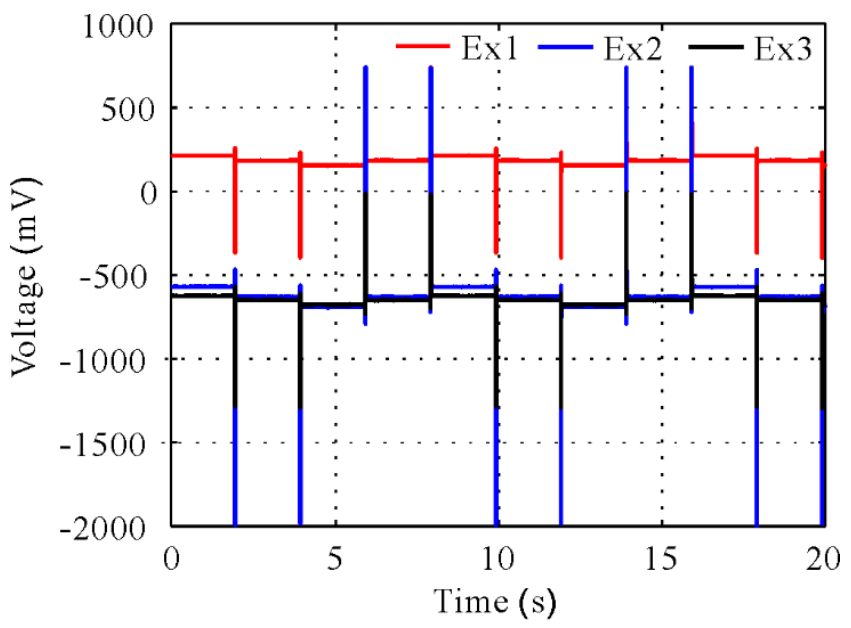

Fig. 17. Raw data of the field test. The curves of red, blue, and black represent the results obtained by the data collected by channel 1, channel 2, and channel 3 (Ex1, Ex2, and Ex3, with the electrodes parallel with each other).

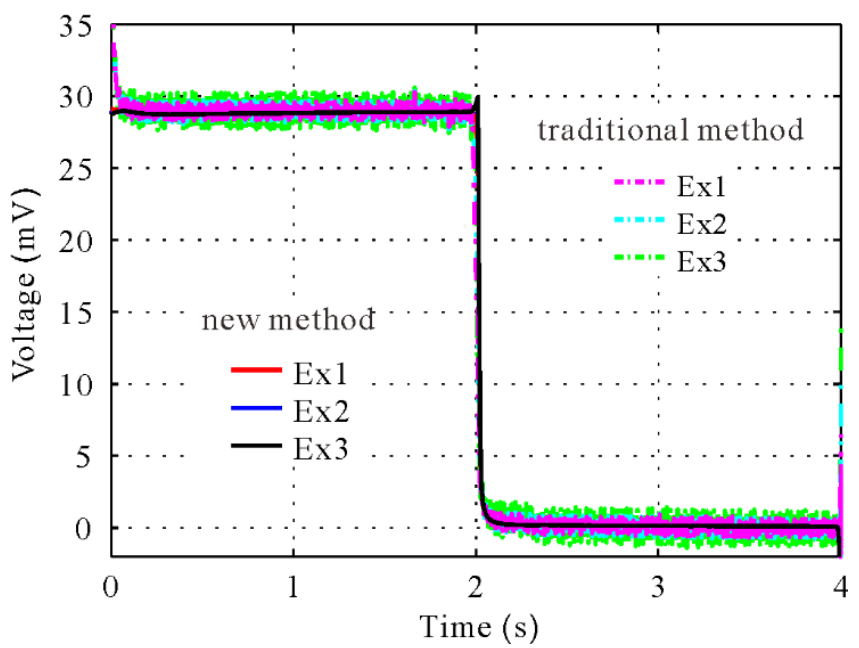

Fig. 18. Decay curve of the field test. The solid curves were calculated by the new method and the dotted curves were analogue results of the traditional method respectively. Ex1, Ex2, and Ex3 represented the results obtained by the data collected by channel 1, channel 2, and channel 3.

(2010). But fitting criterions were different. In our algorithm, the primary field and the secondary field were processed by cubic spline interpolation and tendency fitting separately, and fitting lengths were twice of the overcharging. At the same time, the self-potential was rather strong, but the power 


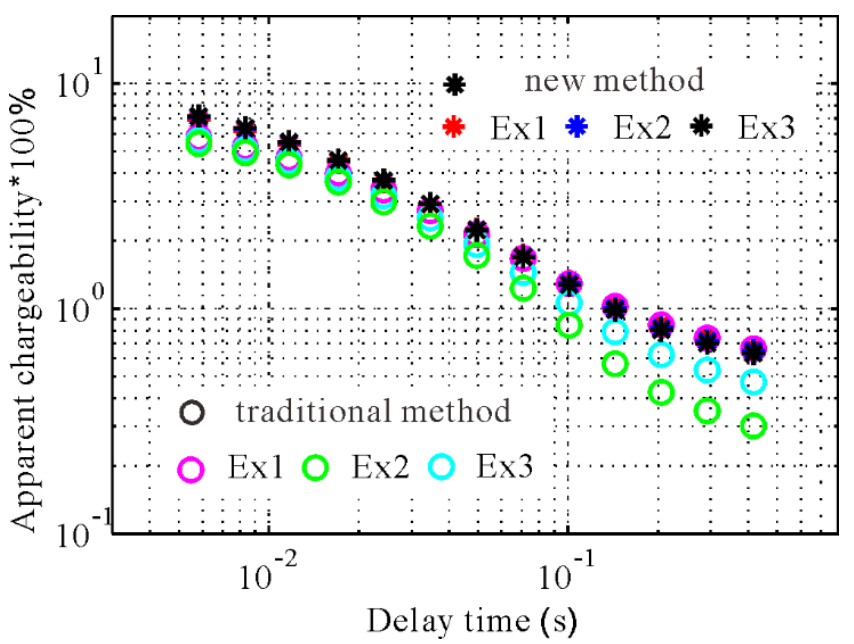

Fig. 19. Apparent chargeability of the field test. Symbols: results obtained by using traditional method (o) and the data-processing algorithm proposed in this paper $(*)$, respectively.

line interference was negligible. So the filtering step was overleaped in the following data processing. Traditional method and the new method without notch filter were used. The curve fitting was also used for both of them to improve the readability of the results. The decay curve and apparent chargeability were shown in Figs. 18 and 19.

\section{DISCUSSION}

For the simulation test, by analyzing the time domain and frequency domain wave of the raw data and the filtered data, both of the two methods have successfully removed the power line interference. By comparison, sinusoid subtraction has preserved the remaining spectral content of the signal, including the frequencies close to the power line interference; moreover, it has the advantages of early stability and no phase delay. But the interference attenuation is obviously lower than that brought by the notch filter. Indeed, the attenuation distortion at frequencies in the vicinity of the notch did not affect the TDIP result directly, but insufficient interference suppression did. As a result, both the decay curve and the apparent chargeability obtained by using the digital notch filter were closer to the theoretical values of the model than by using sinusoid subtraction. Hence, the results obtained by the non-filtered data were seriously distorted.

In the lawn experiment, conventional method based on time control was fail to obtain good results in the case of a synchronization failure and the en- 
vironment interference, but the new algorithm described in Section 3 worked well and valid IP parameters were obtained.

As for the field test, the difference of the raw data collected by the three channel were disturbed by the SP and the inconsistency of channels. In addition, the time-domain data were filled with spikes induced by over-charging. Nevertheless, effective decay curves and apparent chargeability were obtained by the new algorithm, with the results of different channels almost consistent with each other. In contrast, there were obvious differences among the IP results of different channels calculated by the traditional method, and the result of channel 1 which had the minimum self-noise was closest to the new algorithm's results.

\section{CONCLUSIONS}

To address various problems existing in TDIP measurement, including power line interference, incomplete decay curves, and synchronous technology limitations, an improved algorithm has been proposed here to increase the interference rejection capability and obtain more complete information on the secondary field. The first step was to design an effective digital notch filter for power line interference which is available for multiple dataprocessing fields and to code it in $\mathrm{C}++$ and MATLAB separately. In addition, a hardware model of this filter was constructed with $f_{s}=2400 \mathrm{~Hz}$ and $f_{0}=50 \mathrm{~Hz}$ in DSP Builder, and an IP core was obtained. Next, segmentation, overlapping, and averaging methods were used to suppress the random noise and remove SP. As part of this process, the "shifting average difference method" was proposed to determine the time range of the secondary field. By this method, it was possible to determine exactly the jump edge of the secondary field in the case of incomplete synchronization of the receiver and the transmitter. Finally, a series of tests verified that this method can obtain approximately complete secondary field information and apparent chargeability at different decay times in a powerful noise environment. The notch filter can greatly improve the SNR and is suitable for TDIP measurement. The agreement of the calculated and theoretical results proved the effectiveness and accuracy of the notch filter and the algorithm. Indoor and field tests indicated that the new algorithm described in Section 3 could improve the anti-interference ability of TDIP method significantly, and it was also help to overcome the restriction of the adverse field conditions and the effect of the instruments' inherent differences. By using this algorithm, we can get a complete decay curve as well as general TDIP parameters from the raw data which was seriously polluted by various interferences.

Acknowledgments. This research is sponsored by 863 Program (2012AA09A201), National Natural Science Foundation of China 
(61531001), and the open fund (No. GDL1411) of the Key Laboratory of Geo-detection (China University of Geosciences, Beijing), Ministry of Education. We thank MathWorks for the MATLAB code used for filter design and simulation. We are truly grateful to the editors' and reviewers' advices and suggestions on this paper.

\section{References}

Butler, K.E., and R.D. Russell (1993), Subtraction of powerline harmonics from geophysical records, Geophysics 58, 6, 898-903, DOI: 10.1190/1.1443474.

Butler, K.E., and R.D. Russell (2003), Cancellation of multiple harmonic noise series in geophysical records, Geophysics 68, 3, 1083-1090, DOI: 10.1190/ 1.1581080 .

Dahlin, T., V. Leroux, and J. Nissen (2002), Measuring techniques in induced polarisation imaging, J. Appl. Geophys. 50, 3, 279-298, DOI: 10.1016/S09269851(02)00148-9.

Deng, M., and Z.X. He (1998), Data Acquisition characteristics and data acquisition system of differentially-normalized method, Geoscience - J. Graduate School Chin. Univ. Geosc. 12, 3, 442-446 (in Chinese).

Deng, M., F. Liu, Q. Zhang, and K. Chen (2006), Long-span and multi-point synchronizing data acquisition for seafloor magnetotelluric based on union of marine and land, Sci. Technol. Rev. 24, 10, 28-32 (in Chinese).

Deng, M., W. Wei, Q. Zhang, and M. Wang (2008), Real-time backup technique for reducing data loss in seafloor magnetotelluric data acquisition, J. Cent. South Univ. (Science and Technology) 39, 2, 362-367 (in Chinese).

Dhillon, S.S., and S. Chakrabarti (2001), Power line interference removal from electrocardiogram using a simplified lattice based adaptive IIR notch filter. In: Proc. 23rd Ann. Int. Conf. IEEE, DOI: 10.1109/IEMBS.2001.1019561.

Fan, S.J., and X.G. Yang (1979), SDJ-1 electric logging device, J. Shandong Eng. Col. 4, 94-104 (in Chinese).

Feng, Y.J., and Z.H. Fu (1994), DJD6-1 multichannel IP instrument, Geosci. Instr. 3, 33-36 (in Chinese).

Fiandaca, G., E. Auken, A.V. Christiansen, and A. Gazoty (2012), Time-domaininduced polarization: Full-decay forward modeling and 1D laterally constrained inversion of Cole-Cole parameters, Geophysics 77, 3, E213-E225, DOI: 10.1190/geo2011-0217.1.

Galiana-Merino, J.J., D. Ruiz-Fernandez, and J.J. Martinez-Espla (2013), Power line interference filtering on surface electromyography based on the stationary wavelet packet transform, Comput. Meth. Prog. Biomed. 111, 2, 338-346, DOI: 10.1016/ j.cmpb.2013.04.022. 
Gazoty, A., G. Fiandaca, J. Pedersen, E. Auken, A. Christiansen, and J. Pedersen (2012), Application of time domain induced polarization to the mapping of lithotypes in a landfill site, Hydrol. Earth Syst. Sci. 16, 6, 1793-1804, DOI: 10.5194/hess-16-1793-201.

Hönig, M., and B. Tezkan (2007), 1D and 2D Cole-Cole-inversion of time-domain induced-polarization data, Geophys. Prospect. 55, 1, 117-133, DOI: 10.1111/j.1365-2478.2006.00570.x.

Hördt, A., R. Blaschek, A. Kemna, and N. Zisser (2007), Hydraulic conductivity estimation from induced polarisation data at the field scale - the Krauthausen case history, J. Appl. Geophys. 62, 1, 33-46, DOI: 10.1016/j.jappgeo.2006. 08.001 .

Hu, G.S. (2003), Digital Signal Processing: Theory, Algorithms and Implementation, 2nd ed., Tsinghua University Press, Beijing, 648 pp. (in Chinese).

Kohno, R., H. Imai, M. Hatori, and S. Pasupathy (1990), An adaptive canceller of cochannel interference for spread-spectrum multiple-access communication networks in a power line. Selected areas in communications, IEEE J. Sel. Area 8, 4, 691-699, DOI: 10.1109/49.54465.

Li, J.H., P.R. Lin, B.L. Xu, Q.K. Meng, and D. Li (2013), Study on multifunctional electromagnetic prospecting technology in mineral exploration, Adv. Mat. Res. 734, 178-182, DOI: 10.4028/www.scientific.net/AMR.734-737.178.

Marshall, D.J., and T.R. Madden (1959), Induced polarization, a study of its causes, Geophysics 24, 4, 790-816, DOI: 10.1190/1.1438659.

Martinho, E., F. Almeida, and M. Senos Matias (2006), An experimental study of organic pollutant effects on time domain induced polarization measurements, J. Appl. Geophys. 60, 1, 27-40, DOI: 10.1016/j.jappgeo.2005.11. 003.

Nimunkar, A.J., and W.J. Tompkins (2007), EMD-based 60-Hz noise filtering of the ECG. In: Proc. 29th Ann. Int. Conf. IEEE EMBS 2007, DOI: 10.1109/ IEMBS.2007.4352688.

Pankratov, O.V., and A.I. Geraskin (2010), On processing of Controlled Source Electromagnetic (CSEM) data, Geol. Acta Int. Earth Sci. J. 8, 1, 31-49, DOI: $10.1344 / 105.000001514$.

Sahambi, J., S. Tandon, and R. Bhatt (1997), Quantitative analysis of errors due to power-line interference and base-line drift in detection of onsets and offsets in ECG using wavelets, Med. Biol. Eng. Comput. 35, 6, 747-751, DOI: 10.1007/BF02510988.

Seigel, H.O. (1959), Mathematical formulation and type curves for induced polarization, Geophysics 24, 3, 547-565, DOI: 10.1190/1.1438625.

Su, M.-L., and K.-S. Chuang (2013), An ECG signal enhancement based on improved EMD. In: PIERS Proceedings, Taipei, Taiwan.

Swift Jr., C. (1973), The L/M parameter of time-domain IP measurements - A computational analysis, Geophysics 38, 1, 61-67, DOI: 10.1190/1.1440334. 
Tong, M., L. Li, W. Wang, and Y. Jiang (2006), A time-domain induced-polarization method for estimating permeability in a shaly sand reservoir, Geophys. Prospect. 54, 5, 623-631, DOI: 10.1111/j.1365-2478.2006.00568.x.

Wan, H., R. Fu, and L. She (2006), The elimination of $50 \mathrm{~Hz}$ power line interference from ECG using a variable step size LMS adaptive filtering algorithm, Life Sci. J. 3, 4, 90-93.

Warden, S., S. Garambois, P. Sailhac, L. Jouniaux, and M. Bano (2012), Curveletbased seismoelectric data processing, Geophys. J. Int. 190, 3, 1533-1550, DOI: $10.1111 / \mathrm{j} .1365-246 X .2012 .05587 . x$.

Winder, S. (2002), Analog and Digital Filter Design, 2nd ed., Newnes, Amsterdam.

Wu, J.G., and H. P. Dou (1996), DCX-1 active electric field differential instrument, Petrol. Inst. 10, 1, 21-24 (in Chinese).

Xia, J., and D. Miler (2000), Design of a hum filter for suppressing power-noise in seismic data, J. Environ. Eng. Geophys. 5, 2, 31-38, DOI: 10.4133/JEEG5. 2.31 .

Zhang, D.J. (2009), Circuit design of late-model IP detection system, M.Sc. Thesis, China University of Geosciences, Beijing, China (in Chinese).

Received 15 June 2015

Received in revised form 2 March 2016

Accepted 21 March 2016 